\title{
Improving the Odds of Compliance: Using a Brief Telephone Call to Decrease Pre-treatment Attrition in Cardiac Rehabilitation
}

\author{
Dean Cooley ${ }^{1, *}$, Karla Kubitz ${ }^{2}$, Scott Pedersen ${ }^{1}$, Andrew Williams ${ }^{3}$ \\ ${ }^{1}$ Human Movement, Faculty of Education, University of Tasmania, Launceston, Tasmania, 7248 Australia \\ ${ }^{2}$ Department of Kinesiology, Towson University, Baltimore, 21252, Maryland United States \\ ${ }^{3}$ School of Human Life Sciences, Faculty of Health Science, University of Tasmania, Launceston, 7248, Australia \\ *Corresponding Author: Cooley@utas.edu.au
}

Copyright (C) 2013 Horizon Research Publishing All rights reserved.

\begin{abstract}
The purpose of this study was to test the effect of a brief telephone intervention based on the principles of motivational interviewing to reduce pre-treatment attrition in an Australian cardiac rehabilitation centre. The study was an intact comparison randomly trial. Twenty-eight adult patients diagnosed with symptoms associated with cardio-vascular disease, who were noncompliant to an initial appointment, were randomized to receive either a standard cue call or a motivational interview-based recruitment call. Compliance was measured as attendance at a booked session at the cardiac rehabilitation center. Results showed that patients who received a motivational based telephone call had 6 times the odds as those who received a standard cue telephone call for attending a booked appointment. Reducing pre-treatment attrition from cardio-vascular rehabilitation centers can be accomplished with a brief telephone intervention, which incorporates the spirit of motivational interviewing..
\end{abstract}

Keywords Motivational Interviewing, Pre-Treatment Attrition, Cardiac Rehabilitation

\section{Introduction}

Regular participation in physical activity is a mechanism that improves overall physical functioning so that individuals can better meet the demands of daily living [1]. People diagnosed with cardiovascular disease (CVD) are often prescribed physical activity as therapy to improve cardiovascular fitness and reduce mortality and morbidity risk [2]. Such therapy often centers on increasing participation in aerobic type activities at recommended dose and frequency levels [3]. This is sound, as there are beneficial personal and community health outcomes [4]. Nonetheless, a notable proportion of persons who would benefit from physical activity interventions fail to start such treatment [5]. Pre-treatment attrition (PTA) is defined as the non-attendance at a first appointment or non-initiation of a treatment [6-7], and is one of the main reasons for poor long-term effectiveness of health interventions [7]. PTA rates in areas such as drug and psychological counseling are reported between $20-57$ percent [6-7]. As such it represents an issue in publically funded health treatments because non-initiation of a treatment jeopardises clients' health and wellbeing, and interferes with practitioners' efforts to help clients achieve improved health outcomes [5].

Reasons for PTA encompass a variety of individual (i.e., age, presence of a disorder), socio-economic (i.e., knowledge, referral status), and environmental factors (i.e., such as trouble getting off work) [6-10]. Interventions designed to address these factors and alleviate PTA have returned modest but promising effect sizes for increasing compliance [10]. The modest effect sizes are encouraging but interventions that address individual motivation about behaviour change might be more effectual [11]. The decision to enter into treatment signifies a decision to change behavior, which in turns reflects individual motivation [12-13]. Such decisions are normally preceded and accompanied by feelings of ambivalence [13]. Feelings of ambivalence can be detrimental to motivation because individuals are likely to engage in procrastination and avoidance. Thus, an intervention that addresses ambivalence might yield promising results for reducing PTA [12].

It is understandable that patients who have adverse health conditions and are prescribed exercise as a therapy might harbour feelings of ambivalence. Ambivalence about health behavior change can be best described as the possession of positive and negative thoughts [13]. For cardiac rehabilitation clients, even though the treatment offers potential relief from morbidity and mortality, patients' perceptions of exercise, founded or unfounded, might generate feelings of apprehension and catastrophe [14]. For example, patients' concerns regarding the effect of exercise on pre-existing conditions, the suitability of exercise with a 
cardiovascular condition, and outcome expectancies all mediate participation [15]. Patients with such negative perceptions of entering an exercise clinic can then have this manifest into feelings of ambivalence. On the one hand, the treatment presents a circumstance that the patient wishes to approach, yet simultaneously, there are conditions that the patient wishes to avoid. Avoidance can then lead to the adoption of behaviors, such as effort withdrawal by not attending booked appointments [5].

The use of motivational interviewing is an effective counseling technique to reducing ambivalence associated with treatment entry [16-17]. Motivational interviewing (MI) is a directive, client-centred counseling style for eliciting behavior change by helping clients to explore and resolve ambivalence [13]. Within the technique of MI, there are several strategies that can be used to address patient ambivalence without having to use the full counseling process [18]. Typically such counseling contains the use of a conversation style that reflects the spirit of MI, and the use of a MI based strategy [18 -20]. Research outcomes indicate that such a counseling style is successful in changing behaviors associated with various chronic conditions (e.g., diabetes, asthma, alcohol abuse) [20, 21], adoption of lifestyle changes (e.g., smoking cessation, physical activity, diet changes) $[16,17,22]$. Moreover, the MI-based counseling style allows counselors to use of variety of delivery methods including telephone interviews to help change behaviour [23]. Nonetheless, there is little evidence to suggest the effectiveness of MI-based strategies in addressing PTA in a cardiac rehabilitation environment.

Pre-treatment attrition in cardiac rehabilitation is problematic because most reports use the term dropout as an all-inclusive statistic, which might include PTA and non-adherence figures. Some studies report dropout rates as high as 61 percent for males and 47 percent for females [15]. The time interval between a cardiac event and rehabilitation influences dropout rates. Rates range from 20 to 25 percent when patients start rehabilitation within three weeks of a cardiac event but soar to 40 to 50 percent when rehabilitation starts six to twelve months after an event [24]. Costs of cardiac rehabilitation programs vary across countries but in Australia a twice-weekly program is costed at approximately $\$ 480$ per patient completing the full program [24]. Given the lack of data surrounding PTA related to cardiac rehabilitation centers and ways of addressing PTA behaviour we formed speculations about how to address a PTA issue at an exercise physiology center (EPC) located at the University of Tasmania.

Managers of the EPC expressed their concern to the researchers about the high rates of non-attendance at first appointments for patients referred to the EPC through the Lifescripts [25] system. Lifescripts is a program in Australia whereby medical practitioners can write prescriptions for their patients to attend publically subsidized exercise clinics with the goal of preventing or alleviating the detrimental effects of various lifestyle diseases. The range of diseases and symptoms that might trigger a patient referral are; onset of type II diabetes, obesity/overweight, hypertension, or high cholesterol. Given the guidelines that telephone calls are a part of best practice guidelines for cardiac rehabilitation centers [24], and previous research to indicate the success of such counseling [23], we elected to use a telephone call intervention to test the efficacy of a telephone protocol using a MI-based counseling strategy to increase patient compliance to attend an EPC appointment. It was hypothesized that participants who received a MI-based recruitment call would have more compliance to attending a booked appointment at an EPC than participants who received a cue call.

\section{Materials and Methods}

\subsection{Participants}

Participants $(N=35)$ were part of a total of 85 patients referred to the EPC during the study period through the Lifescript program [25]. These 35 participants were included in the study because they were noncompliant ( $>30$ days after referral) in attending a first appointment. The managers of the EPC referred names and contact details to the researchers. Of the original noncompliant participants, some $(n=7)$ could not be contacted due to technical or personal issues; incorrect numbers $(n=2)$, disconnected numbers $(n=2)$ or unwillingness to discuss their referral $(n=3)$. The remaining participants (16 men; 12 women) were included in the final analysis. The mean age of the participants was $47 \pm$ 9.8 ) years, with no statistical difference for gender $(p=0.12)$.

All participants in the study were Caucasian, drawn from the urban areas of Launceston, a small city in the state of Tasmania, Australia. Australian Bureau of Statistics [25] reported that Tasmanians exhibit a number of health risk factors, relating to diet, alcohol consumption, body mass index (BMI), exercise level, and smoking status. In 2007-08 approximately three-quarters of adult aged Tasmanians reported being sedentary or having low exercise levels. Over half of adult Tasmanians (64\%) were found to be overweight or obese, with 25 percent of adult Tasmanians defined as current smokers. Diseases of the circulatory system accounted for over one third $(34.2 \%)$ of all deaths in Tasmania in 2007, which accounts for $22.4 \%$ of the state's total burden of disease. This burden is 40 percent higher than the Australian average (16.4\%).

The site for the EPC was located on the University campus, which is 5 kilometres from the center of Launceston (pop. $\sim 100000$ ) people. The EPC site is accessible by all modes of public transport (bus \& taxi) and private transport (car \& bike). All appointments were free of charge and appointments could be individually scheduled. Given the location's accessibility and lack of cost for individuals to attend, most of the logistical and socio-economic factors known to influence PTA were muted for the study. The study was approved by the University of Tasmania Social Sciences Human Research Ethics Committee (Ref No: H9003) in 
terms of the risks associated with exercise programs and allocation to experimental and control groups.

\subsection{Experimental Design}

Two recruitment telephone call conditions were established for the study. In condition 1, participants received a cue telephone call and in condition 2, participants received a MI-based telephone call. The independent variable was the type of telephone call condition (MI-based or cue) delivered to noncompliant participants. The dependent variable was patient compliance. Compliance was operationally defined as attending a re-booked appointment at the EPC. All participants were randomly assigned to one level of the treatment.

\subsection{Materials}

\subsubsection{Cue call}

The call recruitment script directly focused on having the participant make an appointment to attend the EPC. After viewing counselors conducting their standard calls to follow up patients to book an appointment at the EPC, the researchers composed a standard cue call script for the study. The script contained words and phrases which cued participants to: the purpose of the EPC, reasons for referral, clothing requirements, location, transport and parking options, and suitable times for booking an appointment to attend the EPC. The counselors were given the list of words and phrases to use when conducting the cue call.

\subsubsection{MI-based call}

The MI-based call contained an amalgamation of several MI principles and techniques [20]. We adapted existing protocols [23-25] that explored each individual's readiness to begin behavior change. Consistent with the spirit of MI, the counselors began by emphasizing personal choice, moved to an assessment of readiness using the 'readiness ruler', worked with the patient's responses to the 'scaling questions', and concluded by summarizing and reflecting 'change talk'. The initial question centered on readiness: "On a scale of 1 to 10 (with 10 being the highest), how ready are you to start an exercise program?" The counselors then followed with two probes: (1) "why not a lower number?" and then: (2) "what would it take for you to go to a higher number?" to elicit barriers and strengths associated with the behavior change. The counselors then moved into a summary of the participant's reasons for wanting or not wanting to attend the EPC clinic and asked if there was anything else the participant wanted to add. In this phase of the call, the counselor used the probe: "So, you feel..." If barriers were presented, the counselors prompted the participant to offer solutions. If participants exhausted their own solutions (or if none were offered), the counselors sought permission to list other solutions. When appropriate, the encounter ended with request to re-book an appointment.

\subsection{Procedures}

Counselors were all trained in using recruitment calls based on MI-based strategies but were non-clinical staff. This training of three-hours duration consisted of understanding and educating in the spirit of MI conversations, the use of reflective listening, and the use of the ruler strategy. All counselors were observed performing MI-based calls $(n=2)$ during the study to ensure they complied with the protocols and adhered to the scripts. Counselors were given a list of names and telephone numbers. Associated with each name was an identifier for which script was to be used for each participant. For each script, the counselors were instructed to enter into some preamble conversation with participants to establish rapport and when comfortable, to start the script proceedings.

Counselors were instructed to note the number of calls needed to establish contact, particulars of contact or non-contact (i.e., incorrect telephone numbers), and when contact was established, start and end call times. At the end of each conversation counselors made an appointment by recording the date and time of each in an electronic booking system. These appointments were then transmitted to EPC staff. EPC staff were blind to the intervention conditions. EPC staff then made note of participants' compliance in the booking system. Notation by EPC staff indicated for each participant whether they kept their appointment or did not attend. Researchers then collated the compliance data from the booking system.

\subsection{Analyses}

Consistent with previous research that examines the effectiveness of health treatments [26], compliance data were analyzed using the odds ratio [26, 27]. Confidence intervals (95\%) were calculated, with the proposed MI intervention deemed successful if the odds ratio was greater than one and the lower and upper bound of the confidence interval did not contain one [26]. All statistical analysis were calculated using MedCalc [28].

\section{Results}

Before the study period there was a high rate of PTA (41\%) between doctor referral and appointment booking. For the intervention with the noncompliant cohort, the mean duration for the MI-based call was 38 minutes, with a range between 17 and 47 minutes. The mean duration for the cue call was 12 minutes, with a range of 3 to 27 minutes. Moreover, on average for both telephone conditions, almost three telephone calls were needed to establish contact with participants (i.e., participant not in attendance, inconvenient time). In terms of approximate costs for the intervention, using a standard rate of pay for a research assistant $(\$ 37.55$ per hour) and the estimate call times (mean $\mathrm{X}$ number of calls) for each condition, the total costs of the intervention 
for the cue call intervention was approximately $\$ 309$ ( $\$ 37.55$ X 8.23 hours.), whereas the MI-based call intervention was slightly higher at $\$ 365$ (\$37.55 X 9.5 hours).

There was an effect for the primary outcome of compliance consistent with the hypothesis. Participants who were non-contactable were not included in the analysis. Overall, 66 percent in the MI-based call condition (odds $=2$ ) and 38 percent in the cue call condition ( $o d d s=0.3$ ) made and turned up for a booked appointment at the EPC. Those in the MI-based call condition were more likely to make a behavioural change than those in the standard cue call condition, odds ratio $=6.6($ CI 95\% $1.24-35.71, p=0.02)$.

Table 1. Compliance to booked appointment at an exercise physiology clinic for cardiac rehabilitation after telephone recruitment.

\begin{tabular}{cccc}
\hline Group & Compliant & Non-compliant & Total \\
\hline $\begin{array}{c}\text { MI-based telephone } \\
\text { condition }\end{array}$ & 10 & 5 & 15 \\
$\begin{array}{c}\text { Cue call telephone } \\
\text { condition }\end{array}$ & 3 & 10 & 13 \\
Non-contactable & 1 & 6 & 7 \\
\hline Total & 14 & 21 & 35 \\
\hline
\end{tabular}

In terms of changing behaviour, participants who received a MI-based telephone call were nearly 7 times the odds as participants who received a standard cue call, of attending the booked appointment at the EPC. Thus, the research hypothesis was accepted that a MI-based recruitment telephone call significantly increased compliance to a booked appointment than a cue call.

\section{Discussion}

The researchers in the current study applied a MI-based recruitment telephone call to increase appointment making and keeping to an EPC. Overall, consistent with previous research $[12,16,21]$ there was a positive effect associated with using a MI-based strategy to reduce PTA associated with appointment making and keeping. Non-clinical trained counselors who were familiar with the spirit of MI and who followed a MI strategy according to a script were able to deliver this brief intervention. This result provides evidence that MI-based interventions confer greater benefit than standard cue call procedures.

Consistent with previous research from other medical areas [5,6], we found a high level of PTA within a group of individuals classified as at risk of CVD. The use of physical activity as a preventative therapy to ameliorate CVD is widespread because it is a low cost and non-invasive therapy. Yet, it seems that high percentage of at-risk individuals eschew this treatment. If the result of this study is reflective of actual PTA figures for cardiac rehabilitation across Australia, then the opportunity to improve the quality of peoples' health is being lost. We are unaware of any other data that describes PTA in cardiac rehabilitation programs other than adherence rates. Future research in cardiac rehabilitation centers should differentiate between PTA and non-adherence statistics to further delineate the problem.

The MI-based intervention targeted individual readiness to change and personal resources to bring about the change as a means of addressing ambivalence [13]. It appears that using a MI-based strategy to assess clients' readiness and understanding of their personal resources is an effective mechanism to reduce PTA. Our study finding suggests that an MI-based strategy approach can be achieved by non-clinical trained staff as a means of reducing PTA in other health interventions. Replicating or extending on our approach might have numerous benefits. Addressing PTA is a necessity in all directed forms of health prevention $[5,6]$. Getting people into a first appointment allows health practitioners an opportunity to allay fears about treatment and to correct any misguided notions that clients might have about their health condition and treatment [24]. Moreover, a further benefit is that non-clinical counselors can be used to deliver the intervention thus eliminating the need for clinical trained counselors (i.e., nurses, physiologists, psychologists) from the task of reducing PTA. This would then potentially allow trained counsellors and clinical staff to direct their time to their particular areas of expertise.

Nonetheless, we found that the use of MI-based telephone calls require a greater amount of counselor time in delivering a brief intervention as well as increased costs for the brief intervention compared to a standard call protocol. Similar, a small amount of time needs to be allocated to training and observing counselors to ensure adherence to the script. This time investment might be seen as detrimental but this should be set against the potentiality to limit client lost to an intervention. Moreover, the cost of CVD, both economical and personal, outweight the costs of training non-clinical staff to deliver an effective intervention.

There are limitations to this study. One limitation is the small number of participants and the acute nature of the study. The measure of compliance was appointment making and keeping but there is a need to complete further studies to determine the long-term benefits of using MI during the treatment phases. Other research $[16,22]$ has returned some positive results for the use of MI counseling during treatment. Second, it is unknown which particular aspect of the MI-based ruler strategy was effective in addressing ambivalence. For example, it is unknown if the MI-based call changed participants' feelings of self-efficacy, confidence, or previous experiences of exercise. Understanding which aspects are more salient will allow health professionals to develop more targeted brief interventions. Finally, it is unknown if those who failed to attend the appointment started exercising on their own accord. Future studies should address these issues to ensure that actual mechanisms of MI-based interventions are fully understood.

\section{Conclusion}

Increasing physical activity levels for at risk individuals 
is a cost effective and minimal invasive means of alleviating CVD within the community. Yet, a high percentage of at risk individuals who would benefit from increases in their levels of physical activity choose never start treatment. Ameliorating pre-treatment attrition can be achieved by recruiting participants through the use of a brief telephone intervention that follows the spirit of MI and uses MI strategies. In particular the ruler strategy of MI seems to reduce patient ambivalence about appointment making and keeping. Moreover, non-clinical staff can be easily trained to deliver the intervention to reduce costs associated with the running of cardiac rehabilitation centres.

\section{REFERENCES}

[1] R. Ross, A. McGuire, Incidental physical activity is positively associated with cardiorespiratory fitness, Medicine \& Science in Sports \& Exercise, Vol.44, No.3, 2189-2194, 2012.

[2] P. D. Thompson, D. Buchner, I. L. Piña, G. J. Balady, M. A. Williams, B. H. Marcus, K. Berra, S. N. Blair, F. Costa, B. Franklin, G. F. Fletcher, N. F. Gordon, R. R. Pate, B. L. Rodriguez, A. K. Yancey, N. K. Wenger. Exercise and physical activity in the prevention and treatment of atherosclerotic cardiovascular disease. Circulation, Vol.107, 3109-3116, 2003

[3] I. M. Lee, E. J. Shiroma, F. Lobelo, P. Puska, S. N. Blair, P. T. Katzmarzyk, Effect of physical inactivity on major non-communicable diseases worldwide: an analysis of burden of disease and life expectancy, Lancet, Vol.380, No.9838, 219-229, 2012.

[4] O. H. Franco, C. de Laet, A. Peeters, J. Jonker, J. Mackenbach, W. Nusselder, Effects of physical activity on life expectancy with cardiovascular disease, Archives of Internal Medicine, Vol.165, No.20, 2355-2360, 2005.

[5] K. Simms, C. Myers, J. Adams, J. Hartman, C. Lindsey, M. Doler, J. Suhr, Exercise tolerance testing in a cardiac rehabilitation setting: an exploratory study of its safety and practicality for exercise prescription and outcome data collection. Baylor University Medical Center proceedings, Vol.20, No.4, doi: PMC2014801, 2007.

[6] C. Issakidis, G. Andrews. Pretreatment attrition and dropout in an outpatient clinic for anxiety disorders. Acta Psychiatrica Scandanavica, Vol.109, 426-433, 2004.

[7] C. B. Benway, V. Hamrin, T. J. McMahon. Initial appointment nonattendance in child and family mental health clinics. American Journal of Orthopsychiatry, Vol.73, No.4, 419-28, 2003.

[8] N. L. Lacey, A. Paulman, M. D. Reuter, B. Lovejoy. Why we don't come: Patient perceptions of no-shows. Annuals of Family Medicine, Vol.2, 541-545, 2004.

[9] Benway CB, Hamrin V, McMahon TJ. Initial appointment nonattendance in child and family mental health clinics. American Journal of Orthopsychiatry, Vol.73, No.4, 419-428, 2003.
[10] M. L. Sherman, D. D. Barnum, E. Nyberg, A. Buhman-Wiggs. Predictors of preintake attrition in a rural community mental health center. Psychological Services, Vol.5, No.4, 332-40, 2008.

[11] D. L. Roter, J. A. Hall, R. Merisca, B. Nordstrom, C. Cretin, B. Svarstad. Effectiveness of interventions to improve patient compliance: A meta-analysis. Medical Care, Vol.36, No.8, 1138-61, 1998.

[12] T. Coates, L. Tichter, C. Caceres. Behavioral strategies to reduce HIV transmission: how to make them work better. The Lancet, Vol. 372, No. 9639, 669-684, 2008.

[13] D. Slagle, M. J. Gray. The utility of motivational interviewing as an adjunct to exposure therapy in the treatment of anxiety disorders. Professional Psychology: Research and Practice, Vol.38, No.4, 329-37, 2007.

[14] W. R. Miller, S. Rollnick. Motivational Interviewing, The Guilford Press, London, 1991.

[15] S. Rollnick, W. R. Miller, C. C. Butler. Motivational Interviewing in Health Care: Helping Patients Change Behavior, The Guilford Press, New York, 2008.

[16] C. Bulley , M. Donaghy, S. Howden, L. Salisbury, S. Whiteford, E. Mackay. A prospective qualitative exploration of views about attending pulmonary rehabilitation. Physiotherapy Research International, Vol.14, No.3, 181-93, 2009.

[17] A. M. Clark, K. M. king-Shier, M. A. Spaling, A. S. Duncan, J. A. Stine, S. B. Jaglal, D. R. Thompson, J. E. Angus. Factors influencing participation in cardiac rehabilitation programmes after referral and initial attendance: qualitative systematic review and meta-synthesis. Clinical Rehabilitation, Vol. 24, 2013, doi: 10.1177/0269215513481046

[18] A. H. Anshel, M. Kang. Effectiveness of motivational interviewing on changes in fitness, blood lipids, and exercise adherence of police officers: An outcomes-based action study. Journal of Correctional Health Care, Vol.4, 48-62, 2008.

[19] S. Rubak, A. Sandbaek, T. Lauritzen, B. Christensen. Motivational interviewing: A systematic review and meta-analysis. The British Journal of General Practice: The Journal of the Royal College of General Practitioners, Vol.55, No.513, 305-312, 2005.

[20] B. L. Burke, C. W. Dunn, D. C. Atkins, J. S. Phelps. The emerging evidence base for motivational interviewing: A meta-analytic and qualitative inquiry. Journal of Cognitive Psychotherapy, Vol.18, No.4, 309-22, 2004.

[21] C. Dunn, L. Deroo, E. P. Rivara. The use of brief interventions adapted from motivational interviewing across behavioral domains: a systematic review. Addiction, Vol.961, 725-42, 2001.

[22] S. Rollnick, C. C. Butler, N. Stott. Helping smokers make decisions: the enhancement of brief intervention for general medical practice. Patient Education and Counseling, Vol.31, 191-203, 1997.

[23] C. Butler, S. Rollnick, D. Cohen, M. Bachman, I. Russell, N. Stott. Motivational consulting versus brief advice for smokers in general practice: a randomized trial, British Journal of General Practice, Vol.49, 611-616, 1999.

[24] K. Resnicow, A. Jackson, T. Wang, K. Anindy, F. McCarty, 
W. N. Dudley, T. Baranowski. A motivational interviewing intervention to increase fruit and vegetable intake through black churches: Results of the Eat for Life Trial. American Journal of Public Health, 2001, Vol.91, No.10, 1686-1693, 2001.

[25] McKay JR, Lynch KG, Shepard DS. The effectiveness of telephone-based continuing care for alcohol and cocaine dependence. Archives of General Psychiatry. 2005;62:199-207.

[26] A. B. Goble, M. U. C. Worchester. Best practice guidelines for cardiac rehabilitation centres and secondary prevention. The Heart Researtch Centre, Department of Human Resources, Victoria,1999. R. Ross, A. McGuire, Incidental physical activity is positively associated with cardiorespiratory fitness, Medicine \& Science in Sports \& Exercise, Vol.44, No.3, 2189-2194, 2012.
[27] Department of Health and Aging. Lifescripts. Australian Federal Government. Canberra:, 2008, Available from http://www.health.gov.au/lifescripts

[28] Australian Bureau of Statistics. Tasmanian State and Regional Indicators, March 2010.http://www.abs.gov.au/AU SSTATS/abs@.nsf/Lookup/1307.6 Main+Features13Mar+2010

[29] D. J. Sheskin, Handbook of parametric and nonparametric statistical procedures. 3rd ed. Boca Raton: Chapman \& Hall /CRC, 2004.

[30] J. M.Bland, D. G. Altman. The odds ratio. British Medical Journal, Vol. 320:1468, 2000

[31] MedCalc Software Version 12.7.0, Acacialaan 22, B-8400 Ostend, Belgium, 2013. 